ANNALES

POLONICI MATHEMATICI

$82.1(2003)$

\title{
Equilibria and strict equilibria of multivalued maps on noninvariant sets
}

\author{
by Pierre Cardaliaguet (Brest), \\ Grzegorz Gabor (Brest and Toruń) and Marc Quincampoix (Brest)
}

\begin{abstract}
This paper is concerned with existence of equilibrium of a set-valued map in a given compact subset of a finite-dimensional space. Previously known conditions ensuring existence of equilibrium imply that the set is either invariant or viable for the differential inclusion generated by the set-valued map. We obtain some equilibrium existence results with conditions which imply neither invariance nor viability of the given set. The problem of existence of strict equilibria is also discussed.
\end{abstract}

1. Introduction. The problem of finding an equilibrium of a multivalued map $F$ in a set $K \subset \mathbb{R}^{n}$, i.e., a point $x \in K$ such that $0 \in F(x)$, is important in many topics of analysis. Let us only underline that this problem contains the fixed point problem.

Results on existence of such equilibria have already been obtained for instance

- in [10] for $K$ convex compact and $F$ upper semicontinuous with closed convex values,

- in [3] for $K$ a compact $L$-retract and $F$ upper semicontinuous with closed convex values.

(Note that these papers and their bibliography concerns spaces more general than $\mathbb{R}^{n}$.)

We emphasize that conditions used in [10], [3], [11] and [9] imply (at least in $\mathbb{R}^{n}$ ) that $K$ is viable for the differential inclusion

$$
\dot{x}(t) \in F(x(t)) \quad \text { for a.e. } t \geq 0,
$$

namely: For each $x_{0} \in K$ there is at least one trajectory $x(\cdot)$ for (1) with $x(0)=x_{0}$ such that $x(t) \in K$ for all $t \geq 0$. Indeed, these conditions imply

2000 Mathematics Subject Classification: Primary 54C60; Secondary 34A60, 54C65, $47 \mathrm{H} 04$.

Key words and phrases: equilibrium, strict equilibrium, viability, differential inclusion, multivalued map, Lipschitz selection. 
the tangential condition

$$
\forall x \in K, \quad F(x) \cap T_{K}(x) \neq \emptyset
$$

(with $T_{K}(x)$ the usual contingent cone), which is equivalent by the viability theorem (cf. [1]) to the viability of $K$ for (1). This fact is even a key point in the existence of equilibria (cf. [9] or [1, Theorem 3.7.5]).

Here, we propose a different approach. We give various sufficient conditions for equilibrium existence and these conditions do not imply the viability of $K$.

In the present paper, we study the equilibrium problem by looking at topological properties of the subset $K_{s}$ of the boundary of $K$ where all trajectories of (1) leave $K$ immediately. This set is used in the Ważewski topological principle (cf. [6], [5]). Our first main result is the following:

TheOREM 1.1. Let $K \subset \mathbb{R}^{n}$ and $F: \mathbb{R}^{n} \multimap \mathbb{R}^{n}$ satisfy:

(I) $K$ is a compact $C^{1,1} n$-manifold with boundary;

(II) $F$ is a continuous map with compact convex values and at most linear growth;

(III) $K_{s}(F)$ is closed and, if nonempty, it is a $C^{1,1}(n-1)$-submanifold of $\partial K$ with boundary;

(IV) $\chi\left(K, K_{s}(F)\right) \neq 0$.

Then there is an equilibrium of $F$ in $K$.

The key point of our proof is a reduction to an ordinary differential equation via a suitable single-valued approximation of $F$ well adapted to $K_{s}$. The single-valued case, where the map generates a flow, has been studied in [15].

Also we consider topological properties of the complement of $K$ and derive the existence on equilibrium. Note that [13] contains results on equilibrium existence when the complement of $K$ has suitable viability properties.

Finally, we study the existence of a strict equilibrium in $K$, i.e., a point $x \in K$ such that $\{0\}=F(x)$, using selection techniques developed for equilibria. The second main result is

Theorem 1.2. Let $K \subset \mathbb{R}^{n}$ and $F$ satisfy assumptions (I), (III), (IV) of Theorem 1.1,

$$
0 \notin \operatorname{Int}_{\text {lin } F(x)} F(x) \quad \text { for every } x \in K \text { with }|F(x)|>0
$$

(where $|F(x)|:=\sup \{|y| \mid y \in F(x)\}$ and $\operatorname{Int}_{\operatorname{lin} F(x)} F(x)$ denotes the relative interior of $F(x)$ in the subspace $\operatorname{lin} F(x) \subset \mathbb{R}^{n}$ spanned by $F(x)$ ), and let $F$ be Lipschitz with $0 \notin F(x)$ for every $x \in \partial K$. Then there exists a strict equilibrium of $F$ in $\operatorname{Int} K$. 
The organization of the paper is as follows. After some preliminaries the third section is devoted to sufficient conditions for solving the equilibrium problem without viability. In the last section, the existence of strict equilibria is studied.

2. Preliminaries. Throughout the paper, by $\operatorname{Int} A, \operatorname{cl} A$ (or $\bar{A}$ ) and $\partial A$ we denote respectively the interior, closure and boundary of a subset $A$ of a metric space $X$. The open ball centred at $x_{0}$ with radius $r$ is denoted by $B\left(x_{0}, r\right)$, and the unit ball $B(0,1)$ by $B_{1}$. We also write $|\cdot|$ for the Euclidean norm. By $d_{M}(x)$ (or $\operatorname{dist}(x, M)$ ) we denote the distance from a point $x$ to a closed set $M$. By the distance between two sets $N, M \subset X$ we mean the number $\operatorname{dist}(N, M):=\inf \{d(x, y) \mid x \in N, y \in M\}$.

In (1) we assume that $F$ is a Marchaud map, i.e., $F$ is upper semicontinuous with compact convex values and at most linear growth. By a solution to the inclusion (1) we mean an absolutely continuous map $x:[0, \infty) \rightarrow \mathbb{R}^{n}$ satisfying (1) almost everywhere. For $x_{0} \in \mathbb{R}^{n}$, we denote by $S_{F}\left(x_{0}\right)$ the set of all solutions to (1) with $x(0)=x_{0}$ (starting from $\left.x_{0}\right)$.

Definition 2.1. We say that a trajectory $x(\cdot)$ for the inclusion (1) is viable in $K$ if $x(t) \in K$ for all $t \geq 0$. A set $K$ is said to be viable under $F$ if for each $x_{0} \in K$ there is at least one trajectory $x(\cdot)$ for (1) which is viable in $K$ and $x(0)=x_{0}$. The viability kernel of $K$ for $F\left(\right.$ written $\left.\operatorname{Viab}_{F}(K)\right)$ is the largest closed subset of $K$ viable under $F$ (possibly empty, in general). Equivalently (see [1, Theorem 4.1.2]), $\operatorname{Viab}_{F}(K)$ is the subset of all initial states such that from each of them starts at least one solution viable in $K$. We say that the set $K$ is invariant under $F$ if all trajectories for (1) starting from $K$ are viable in $K$.

Obviously, for single-valued locally Lipschitz right-hand sides the notions of viable and invariant sets coincide. Later on we shall use the notation $\sigma(F(x)$ ) for a Steiner point of a convex set $F(x)$ (see e.g. [2]). If $F$ is Lipschitz, the map $x \mapsto \sigma(F(x))$ is a Lipschitz selection of $F$.

One defines

$$
T_{K}(x):=\left\{v \in \mathbb{R}^{n} \mid \liminf _{h \rightarrow 0^{+}} \operatorname{dist}(x+h v, K) / h=0\right\},
$$

the Bouligand tangent cone to $K$ at $x$. Viability and invariance conditions can be characterized using Bouligand cones, as follows (see e.g. [1]):

Proposition 2.2. A closed set $K$ is viable under a Marchaud map $F$ if and only if

$$
F(x) \cap T_{K}(x) \neq \emptyset \quad \text { for every } x \in K .
$$

If $F$ is Lipschitz, then a closed set $K$ is invariant under $F$ if and only if

$$
F(x) \subset T_{K}(x) \quad \text { for every } x \in K \text {. }
$$


We introduce the following notations for subsets of the boundary of $K$ :

$$
\begin{aligned}
& K_{s}(F):=\left\{x_{0} \in \partial K \mid \forall x \in S_{F}\left(x_{0}\right): x \text { leaves } K \text { immediately }\right\}, \\
& K_{e}(F):=\left\{x_{0} \in \partial K \mid \exists x \in S_{F}\left(x_{0}\right): x \text { leaves } K \text { immediately }\right\} .
\end{aligned}
$$

When there is no ambiguity, we shall write briefly $K_{s}$ and $K_{e}$.

Note that for $F$ Lipschitz continuous, using the local invariance theorem (see e.g. [1, Theorem 5.3.4]), one can describe $K_{e}$ by suitable tangent cones conditions. Indeed, if $K_{e}$ is closed, we have

$$
\partial K \backslash K_{e}=\left\{x \in \partial K \mid \exists U_{x} \subset \partial K \text {, open, } x \in U_{x}, \forall z \in U_{x}: F(z) \subset T_{K}(z)\right\} .
$$

Moreover, $K_{e}=K_{s}$ when $F$ is Lipschitz and single-valued.

In the Appendix we also give a necessary and a sufficient condition for points in $\partial K$ to belong to $K_{s}$, in terms of tangent cones. These conditions were first proved in [5] and announced in [6].

Recall that a subset $M$ of $\mathbb{R}^{n}$ is said to be a proximal retract (see e.g. [16]), if there is an open neighbourhood $V$ of $M$ such that

$$
\pi_{M}(x):=\left\{y \in M|| y-x\left|=\inf _{u \in M}\right| u-x \mid\right\} \quad \text { is a singleton }
$$

for every $x \in U$. This means that $\pi_{M}$ is a retraction from $V$ onto $M$. One can prove that each $C^{1,1}$ manifold is a proximal retract.

A subset $M$ of a metric space $X$ is an $\mathcal{L}$-retract (of $X$ ) if there are an open neighbourhood $U$ of $M$ in $X$, a retraction $r: U \rightarrow M$ and a constant $L>0$ such that

$$
d(r(x), x) \leq L \operatorname{dist}(x, M) \quad \text { for every } x \in U .
$$

It is seen that each proximal retract is an $\mathcal{L}$-retract with constant 1.

We say that a set $K$ is of finite type if the graded vector space $\left\{H_{q}(K)\right\}_{q \geq 0}$ is of finite type, i.e., $H_{q}(K)=0$ for almost all $q \geq 0$, and $\operatorname{dim} H_{q}(K)<\infty$ for all $q \geq 0$. Here $H$ denotes the Čech homology functor.

For each set of finite type the Euler characteristic

$$
\chi(K):=\sum_{q=0}^{\infty}(-1)^{q} \operatorname{dim} H_{q}(K)
$$

is defined (see e.g. [4]). If we have a pair $(K, M)$ of spaces ( $M$ closed in $K)$, with $M \subset K$ and such that both $K$ and $M$ are of finite type, we can define $\chi(K, M):=\chi(K)-\chi(M)$. Note that $\chi(K)=\lambda($ id $)$, the Lefschetz number of the identity map.

We will say that a compact space $X$ is a Lefschetz set if for any continuous map $f: X \rightarrow X$, the condition $\lambda(f) \neq 0$ implies that there is a fixed point of $f$. This class of spaces is large. It contains compact absolute neighbourhood retracts and, more generally, compact approximate absolute neighbourhood retracts (see e.g. [14]). 


\section{Equilibria without viability}

3.1. Using properties of $K_{s}$. In this subsection we deal with the situation where $K$ may not be a viability domain. The boundary $\partial K$ may contain simultaneously points at which a field is strictly inward and others where it is strictly outward.

The first result is a simple consequence of the following.

Proposition 3.1 (adapted version of Theorem 4.1 of [15]). Let $f: \mathbb{R}^{n} \rightarrow$ $\mathbb{R}^{n}$ be a Lipschitz map and suppose that $K$ and $K_{s}(f)$ satisfy assumptions (I) and (III) of Theorem 1.1 with $\chi\left(K, K_{s}(f)\right) \neq 0$. Then there is an equilibrium of $f$ in $K$.

As a consequence one obtains the following easy

Corollary 3.2. Let $K \subset \mathbb{R}^{n}$ be a compact subset, $\Omega \subset \mathbb{R}^{n}$ an open neighbourhood of $K$ and $F: \Omega \multimap \mathbb{R}^{n}$ a Lipschitz map. Assume that $K_{s}$ and $K$ are as in Proposition 3.1 and that $K_{s}=K_{e}$. Then there is an equilibrium of $F$ in $K$.

To prove the above corollary, take any Lipschitz selection $f$ of $F$ and apply Proposition 3.1.

Above we have assumed that $F$ is so regular that it has Lipschitz selections for which, under $K_{s}=K_{e}$, we can use a single-valued approach. Weakening these two assumptions, i.e. lipschitzeanity of $F$ and equality $K_{s}=K_{e}$, is the aim of our main Theorem 1.1. In the proof we will use the following crucial lemma.

Lemma 3.3. Let $K$ and $F$ satisfy (I)-(III) of Theorem 1.1 and suppose $0 \notin F(x)$ for every $x \in \partial K$. Then, for every $\varepsilon>0$, there exists $f: \mathbb{R}^{n} \rightarrow \mathbb{R}^{n}$ such that

(a) $\left\langle f(x), \nu_{x}\right\rangle<0$ for every $x \in \partial K \backslash K_{s}(F)$, where $\nu_{x}$ is an outward normal vector to $K$ at $x$;

(b) for every $x \in K$, if $f(x)=0$, then there is $y \in K \cap B(x, \varepsilon)$ such that $0 \in F(y)$;

(c) $K_{s}(f)=K_{s}(F)$;

(d) $f$ is Lipschitz.

Proof. We proceed in two steps.

STEP 1. We construct an open set $U$ in $\mathbb{R}^{n}$ such that $K \backslash K_{s}(F) \subset U$ and $K_{s}(F) \cap U=\emptyset$, and a map $g: U \rightarrow \mathbb{R}^{n}$ such that

(A) $\left\langle g(x), \nu_{x}\right\rangle<0$ for every $x \in \partial K \backslash K_{s}(F)$;

(B) $g$ is $C^{\infty}$ in $U$;

(C) $g(x) \in F(x)+d_{K_{s}(F)}(x) B_{1}$ for every $x \in K \backslash K_{s}(F)$; 
(D) for every $x \in K \backslash K_{s}(F)$, if $0 \notin F(y)$ for each $y \in B(x, \varepsilon) \cap K$, then $g(x) \in F(x)+\frac{1}{2} d_{F(x)}(0) B_{1}$.

To do this, we first find, for every $x \in K \backslash K_{s}(F)$, a vector $v_{x} \in \mathbb{R}^{n}$ and an open neighbourhood $U_{x}$ of $x$ in $\mathbb{R}^{n}$ satisfying the following three conditions:

$$
\begin{gathered}
U_{x} \cap K_{s}(F)=\emptyset, \\
\forall y \in U_{x} \cap K, \quad v_{x} \in\left(F(y)+d_{K_{s}(F)}(y) B_{1}\right) \cap\left(F(y)+\frac{1}{2} d_{F(y)}(0) B_{1}\right), \\
\forall x \in \partial K \backslash K_{s}(F) \forall y \in U_{x} \cap \partial K, \quad\left\langle v_{x}, \nu_{y}\right\rangle<0 .
\end{gathered}
$$

Case 1. Let $x \in \partial K \backslash K_{s}(F)$. Then there exists $w_{x} \in F(x) \cap T_{K}(x)$. Set $\eta_{x}:=d_{K_{s}(F)}(x)>0, \mu_{x}:=d_{F(x)}(0)>0$ and $\varepsilon_{x}:=\frac{1}{8} \min \left\{\mu_{x}, \eta_{x}\right\}$. Take an open neighbourhood $U_{x} \subset B(x, \varepsilon)$ of $x$ such that $d_{K_{s}(F)}(y) \geq \eta_{x} / 2$, $d_{F(y)}(0) \geq \mu_{x} / 2$ and $F(x) \subset F(y)+\varepsilon_{x} B_{1}$ for every $y \in U_{x}$.

Now take $v_{x}:=w_{x}-t_{x} \nu_{x}\left(t_{x}>0\right.$ sufficiently small) such that $v_{x} \in$ $F(x)+\varepsilon_{x} B_{1}$. Then, for $y \in U_{x} \cap K$,

$$
v_{x} \in F(y)+2 \varepsilon_{x} B_{1} \subset F(y)+\frac{1}{4} \eta_{x} B_{1} \subset F(y)+\frac{1}{2} d_{K_{s}(F)}(y) B_{1} .
$$

Analogously,

$$
v_{x} \in F(y)+2 \varepsilon_{x} B_{1} \subset F(y)+\frac{1}{4} \mu_{x} B_{1} \subset F(y)+\frac{1}{2} d_{F(y)}(0) B_{1} .
$$

Moreover, we can take $U_{x}$ so small that $\left\langle v_{x}, \nu_{y}\right\rangle<0$ for every $y \in U_{x} \cap \partial K$, since $\left\langle v_{x}, \nu_{x}\right\rangle=\left\langle w_{x}, \nu_{x}\right\rangle-t_{x}<0$.

Case 2. Let $x \in \operatorname{Int} K$ and $0 \notin F(x)$. As above, we can find $U_{x} \subset B(x, \varepsilon)$ with $U_{x} \cap \partial K=\emptyset$ and $v_{x} \in F(x)$ satisfying (6) and $0 \notin F(y)$ for any $y \in U_{x}$.

Case 3. For $x \in \operatorname{Int} K$ with $0 \in F(x)$, take $v_{x}=0$ and choose $U_{x} \subset$ $B(x, \varepsilon)$ such that $U_{x} \cap \partial K=\emptyset$ and $v_{x} \in F(y)+d_{K_{s}(F)}(y) B_{1}$ for every $y \in U_{x}$.

Choose a countable, locally finite covering $\left\{U_{x_{i}} \mid x_{i} \in K \backslash K_{s}(F)\right\}$ of $K \backslash K_{s}(F)$ and consider a smooth $\left(C^{\infty}\right)$ partition of unity $\left\{\lambda_{i}: U_{x_{i}} \rightarrow[0,1]\right\}$ subordinate to it. Define $U:=\bigcup_{i=1}^{\infty} U_{x_{i}}$ and notice that $K \backslash K_{s}(F) \subset U$ and $U \cap K_{s}(F)=\emptyset$. For every $x \in U$, set $I(x):=\left\{i \in \mathbb{N} \mid \lambda_{i}(x) \neq 0\right\}$ and define

$$
g(x):=\sum_{i \in I(x)} \lambda_{i}(x) v_{x_{i}} .
$$

Since each $\lambda_{i}$ is $C^{\infty}$, (B) holds. To verify (A), take $x \in \partial K \backslash K_{s}(F)$ and notice that, by the definition of $v_{x_{i}}$ and $U_{x_{i}}$, for each $i \in I(x)$, one has $x_{i} \in \partial K \backslash K_{s}(F)$ and

$$
\left\langle g(x), \nu_{x}\right\rangle=\sum_{i \in I(x)} \lambda_{i}(x)\left\langle v_{x_{i}}, \nu_{x}\right\rangle<0 .
$$


Now, for $x \in K \backslash K_{s}(F)$ and $i \in I(x)$, because the right-hand side of (6) is convex one deduces that

$$
g(x)=\sum_{i \in I(x)} \lambda_{i}(x) v_{x_{i}} \in F(x)+d_{K_{s}(F)}(x) B_{1},
$$

and $(\mathrm{C})$ is also satisfied. Finally, let $x \in K \backslash K_{s}(F)$ be such that $0 \notin F(y)$ for every $y \in B(x, \varepsilon)$. Then, by $(9), v_{x_{i}} \in F(x)+\frac{1}{2} d_{F(x)}(0) B_{1}$, and hence $g(x) \in F(x)+\frac{1}{2} d_{F(x)}(0) B_{1}$, verifying (D) and ending Step 1.

STEP 2. In order to construct the map $f$ we first define, for $x \in K_{s}(F)$, a map $v_{x}(\cdot)$ and an open neighbourhood $U_{x}$ of $x$ in the following way.

Case 1. Let $x \in \operatorname{Int}_{\partial K} K_{s}(F)$. We know that $\min _{v \in F(x)}\left\langle v, \nu_{x}\right\rangle \geq 0$ and $0 \notin F(x)$. Fix $w_{x} \in F(x)$, so $\left\langle w_{x}, \nu_{x}\right\rangle \geq 0$. Write $\mu_{x}:=d_{F(x)}(0)$ and take an open neighbourhood $U_{x}$ of $x$ in $\mathbb{R}^{n}$ such that $U_{x} \cap \partial_{\partial K} K_{s}(F)=\emptyset, U_{x} \cap \partial K \subset$ $K_{s}(F)$ and $d_{F(y)}(0) \geq \mu_{x} / 2$ for every $y \in U_{x} \cap K$.

Define $v_{x}:=w_{x}+t_{x} \nu_{x}$, where $t_{x}>0$ is so small that $v_{x} \in F(x)+$ $\frac{1}{8} \mu_{x} B_{1}$, and consequently, inclusion (9) holds for every $y \in U_{x} \cap K$. Moreover, $\left\langle v_{x}, \nu_{y}\right\rangle>0$ for $y \in U_{x} \cap K_{s}(F)$.

Case 2. Suppose that $x \in \partial_{\partial K} K_{s}(F)$. Then, since $x \in K_{s}(F)$ which is closed, we know by Lemma 5.2 in the Appendix that there is $v \in F(x) \cap$ $T_{K}(x)$ such that $v \in T_{K_{s}(F)}(x)$. There is an open neighbourhood $V_{x}$ of $x$ in $\mathbb{R}^{n}$ and a $C^{1,1}$ diffeomorphism $\phi: V_{x} \rightarrow \phi\left(V_{x}\right) \subset \mathbb{R}^{n}$ such that $\phi(x)=0$,

$$
\begin{aligned}
\phi\left(V_{x} \cap K\right) & =\left\{\left(y_{1}, \ldots, y_{n}\right) \in \mathbb{R}^{n} \mid y_{1} \leq 0\right\}=: X, \\
\phi\left(V_{x} \cap K_{s}(F)\right) & =\left\{\left(y_{1}, \ldots, y_{n}\right) \in X \mid y_{1}=0 \text { and } y_{2} \geq 0\right\}=: X_{s} .
\end{aligned}
$$

As a consequence, for $z \in V_{x} \cap K$,

$$
\begin{aligned}
\operatorname{Int} \phi^{\prime}(z)\left(T_{K}(z)\right) & =\left\{\left(y_{1}, \ldots, y_{n}\right) \in X \mid y_{1}<0\right\}, \\
\operatorname{Int} \phi^{\prime}(z)\left(T_{\mathbb{R}^{n} \backslash K}(z)\right) & =\left\{\left(y_{1}, \ldots, y_{n}\right) \in \mathbb{R}^{n} \mid y_{1}>0\right\},
\end{aligned}
$$

and for $z \in V_{x} \cap \partial_{\partial K} K_{s}(F)$,

$$
\operatorname{Int}_{\partial X} \phi^{\prime}(z)\left(T_{K_{s}(F)}(z)\right)=\left\{\left(y_{1}, \ldots, y_{n}\right) \in X \mid y_{1}=0 \text { and } y_{2}>0\right\} \text {. }
$$

Define on $\phi\left(V_{x}\right)$ the map $G(y):=\phi^{\prime}\left(\phi^{-1}(y)\right) F\left(\phi^{-1}(y)\right)$.

Let $\zeta(y):=\left(y_{2}, 1,0, \ldots, 0\right)$ and take $w:=\phi^{\prime}(x) v$. Then $\left\langle w, e_{1}\right\rangle=0$ and $\left\langle w, e_{2}\right\rangle \geq 0$, where $e_{1}, e_{2}$ are the unit vectors of the first two axes. Let $u_{\theta}(y):=w+\theta \zeta(y)$, where $\theta>0$. Then

$$
\begin{array}{ll}
\left\langle u_{\theta}(y), e_{1}\right\rangle<0 & \text { for } y \in \partial X \backslash X_{s}, \\
\left\langle u_{\theta}(y), e_{1}\right\rangle>0 & \text { for } y \in \operatorname{Int}_{\partial X} X_{s}, \\
\left\langle u_{\theta}(y), e_{2}\right\rangle>0 & \text { for } y \in \partial_{\partial X} X_{s} .
\end{array}
$$

Define, for $z \in V_{x}, v_{\theta}(z):=\left(\phi^{-1}\right)^{\prime}(\phi(z)) u_{\theta}(\phi(z))$. Using (13) and the fact that tangent cones correspond to tangent cones under the diffeomorphism 
$\phi($ see $(10)-(12))$, we obtain

$$
\begin{array}{ll}
\left\langle v_{\theta}(z), \nu_{z}\right\rangle<0 & \text { for } z \in \partial K \backslash K_{s}(F), \\
\left\langle v_{\theta}(z), \nu_{z}\right\rangle>0 & \text { for } z \in \operatorname{Int}_{\partial K} K_{s}(F), \\
\left\langle v_{\theta}(z), n_{z}\right\rangle>0 & \text { for } z \in \partial_{\partial K} K_{s}(F),
\end{array}
$$

where $n_{z}$ is an inward normal vector to $K_{s}(F)$ in $T_{\partial K}(z)$.

Similarly to Case 1, we can find an open neighbourhood $U_{x} \subset V_{x}$ of $x$ and a small $\theta_{x}>0$ such that, for $v_{x}(z):=v_{\theta_{x}}(z)$, we have additionally

$$
v_{x}(z) \in F(z)+\frac{1}{2} d_{F(z)}(0) B_{1} \quad \text { for every } z \in U_{x} \cap K .
$$

We have constructed an open covering of $K$ consisting of the $U$ from Step 1 and $\left\{U_{x}\right\}_{x \in K_{s}(F)}$. Since $K_{s}(F)$ is compact, we can choose a finite subcovering $\left\{U_{i}\right\}_{i=0}^{k}$, where $U_{0}:=U$, and consider a smooth partition of unity $\left\{\beta_{i}\right\}$ subordinate to it.

Define

$$
f(x):=\beta_{0}(x) g(x)+\sum_{i=1}^{k} \beta_{i}(x) v_{x_{i}}(x) .
$$

To finish the proof it is sufficient to verify conditions (a)-(d).

To check (a), let $x \in \partial K \backslash K_{s}(F)$ and $I(x):=\left\{i \in\{1, \ldots, k\} \mid \beta_{i}(x) \neq 0\right\}$. From (14) and (A) it follows that $\left\langle v_{x_{i}}(x), \nu_{x}\right\rangle<0$ for $i \in I(x)$, and consequently, $\left\langle g(x), \nu_{x}\right\rangle<0$. Therefore, $\left\langle f(x), \nu_{x}\right\rangle<0$.

Now, suppose that $x \in K$ is such that $0 \notin F(y)$ for every $y \in B(x, \varepsilon)$. Since $v_{x_{i}}(x) \in F(x)+\frac{1}{2} d_{F(x)}(0) B_{1}$ for each $i \in I(x)$, and $g(x) \in F(x)+$ $\frac{1}{2} d_{F(x)}(0) B_{1}$ (see (D)), one obtains $f(x) \in F(x)+\frac{1}{2} d_{F(x)}(0) B_{1}$, and hence $f(x) \neq 0$; condition (b) is satisfied.

To verify (c), notice that $K_{s}(f) \subset K_{s}(F)$ because of (a). Moreover, for $x \in \operatorname{Int}_{\partial K} K_{s}(F), \beta_{0}(x)=0$ and $\left\langle v_{x_{i}}, \nu_{x}\right\rangle>0$ (see Step 2, Case 1). Thus, $\left\langle f(x), \nu_{x}\right\rangle>0$.

If $x \in \partial_{\partial K} K_{s}(F)$, then $x_{i} \in \partial_{\partial K} K_{s}(F)$ for every $i \in I(x)$. Moreover, $\beta_{0}(x)=0,\left\langle v_{x_{i}}(x), \nu_{x}\right\rangle=0$ and $\left\langle v_{x_{i}}, n_{x}\right\rangle>0$, which implies that $\left\langle f(x), n_{x}\right\rangle>0$ while $\left\langle f(x), \nu_{x}\right\rangle=0$. From Lemma 5.2(1) it follows that a trajectory for $f$ starting from $x$ leaves the set $K$ immediately. Therefore $K_{s}(f)=K_{s}(F)$.

Finally, $f$ is Lipschitz by the regularity of $\beta_{i}$ and of the diffeomorphism $\phi$; the proof is complete.

Proof of Theorem 1.1. We can assume that $0 \notin F(x)$ for every $x \in \partial K$. Applying Lemma 3.3 we find a Lipschitz single-valued map $f$ satisfying (a)-(d). Thanks to (IV), we can use Proposition 3.1 for $f$ and obtain its equilibrium $x_{0} \in K$. Property (b) implies that there is also an equilibrium of $F$ in $K$ near $x_{0}$. 
3.2. Using properties of the complement of $K$. Set $\widehat{K}:=\overline{\mathbb{R}^{n} \backslash K}$ and recall that (see [17], [18])

$$
T_{K}(x) \cup T_{\widehat{K}}(x)=\mathbb{R}^{n}, \quad T_{K}(x) \cap T_{\hat{K}}(x)=T_{\partial K}(x) \quad \text { for } x \in \partial K .
$$

The first result using $\widehat{K}$ to study equilibria on $K$, which we would like to present, has been proved in [13] and reads as follows.

Proposition 3.4 ([13, Theorem 3.1]). Let $K=\overline{\operatorname{Int} K} \subset \mathbb{R}^{n}$ be a compact subset of finite type with $\chi(K) \neq 0, \Omega \subset \mathbb{R}^{n}$ an open neighbourhood of $K$ and $F: \Omega \multimap \mathbb{R}^{n}$ a Lipschitz map satisfying

$$
F(x) \subset T_{\widehat{K}}(x) \quad \text { for all } x \in \partial K, \quad \operatorname{Viab}_{F}(K) \cap \partial K=\emptyset .
$$

Then $F$ has an equilibrium in $\operatorname{Int} K$.

REMARK 3.5. Note that condition (16) is clearly satisfied if for all $x \in$ $\partial K, F(x) \cap T_{K}(x)=\emptyset$ or, more generally, if $K_{s}=\partial K$.

Replacing the condition $F(x) \subset T_{\widehat{K}}(x)$ by $F(x) \cap \operatorname{cl}\left(\mathbb{R}^{n} \backslash T_{\widehat{K}}(x)\right)=\emptyset$ narrows the class of problems which may be considered. Indeed, there are situations (see [13]) appropriate for Proposition 3.4 and such that $\operatorname{cl}\left(\mathbb{R}^{n} \backslash T_{\widehat{K}}(x)\right.$ ) $=\mathbb{R}^{n}$ at some points.

Proposition 3.4 together with the above remark allows us to deal with a large class of sets. In [13] there is an example of a set $K$ which is not even an absolute neighbourhood retract and which, together with a Lipschitz single-valued map, satisfies the assumptions of Proposition 3.4.

The goal of this subsection is to weaken assumption (16) to be able to consider also situations where $K$ and $\widehat{K}$ are not invariant under $-F$ and $F$, respectively. We study the behaviour of $F$ with respect to $\widehat{K}$.

We start with the following.

Lemma 3.6. Let $K$ be a Lefschetz set with $\chi(K) \neq 0, \Omega \subset \mathbb{R}^{n}$ an open neighbourhood of $K$ and $f: \Omega \rightarrow \mathbb{R}^{n}$ a Lipschitz map such that $K$ is invariant under $f$. Then $f$ has an equilibrium in $K$.

Proof. It is well known that for $f$ and every $t \geq 0$, the Poincaré operator $P_{t}:=e_{t} \circ S_{f}: K \rightarrow K$, where $e_{t}(x(\cdot)):=x(t)$, is continuous and homotopic to id $: K \rightarrow K$ by the homotopy $H(x, s):=P_{s t}(x)$. Thus

$$
0 \neq \chi(K)=\lambda(\mathrm{id})=\lambda\left(P_{t}\right),
$$

and since $K$ is a Lefschetz set, there is a fixed point $x_{t}$ of $P_{t}$, which means that there is a $t$-periodic trajectory for $f$.

Taking a sequence $t_{n} \rightarrow 0$, one can easily prove that there is a stationary trajectory, which is equivalent to $f$ having an equilibrium. 
We will also use the following simultaneous selection and approximation result.

Lemma 3.7. Let $E$ be a normed space, $X$ a metric space and $A \subset X$ a compact subset. Assume that $F: X \multimap E$ and $\Psi: A \multimap E$ are convex valued, $F$ is u.s.c., $\Psi$ is l.s.c. and the following condition is satisfied:

$$
\begin{aligned}
& \text { for each } x \in A \text { there are } y_{x} \in F(x) \cap \Psi(x) \text { and an open neighbourhood } \\
& U(x) \subset X \text { of } x \text { such that } y_{x} \in \Psi(z) \text { for every } z \in U(x) \cap A \text {. }
\end{aligned}
$$

Then, for every $\varepsilon>0$, there exist an open neighbourhood $\Omega_{\varepsilon}$ of $A$ in $X$ and a Lipschitz map $f: \Omega_{\varepsilon} \rightarrow E$ such that:

(i) $f$ is an $\varepsilon$-approximation of $F$, i.e., $f(x) \in F(B(x, \varepsilon))+\varepsilon B_{1}$ for every $x \in \Omega_{\varepsilon}$,

(ii) $f$ is a selection of $\Psi$ on $A$.

Proof. The idea of the proof is taken from [3]. For a given $\varepsilon>0$, consider the open covering of $A$ in $X$,

$$
U(x):=B(x, \varepsilon / 2) \cap\left\{x^{\prime} \in X \mid F\left(x^{\prime}\right) \subset F(x)+(\varepsilon / 2) B_{1}\right\}, \quad x \in A .
$$

Using (17) we can find, for every $x \in A$, a point $y_{x} \in F(x) \cap \Psi(x)$ and an open neighbourhood $V(x) \subset U(x)$ of $x$ in $X$ such that $y_{x} \in \Psi(z)$ for each $z \in V(x) \cap A$. Since $A$ is compact, we find a finite open star-refinement $\mathcal{V}=\left\{V_{1}, \ldots, V_{k}\right\}$ of $\{V(x)\}_{x \in A}$, i.e., for every $i \in\{1, \ldots, k\}$, there is $\bar{x} \in A$ such that

$$
\operatorname{st}\left(V_{i}, \mathcal{V}\right):=\bigcup\left\{V_{j} \in \mathcal{V} \mid V_{j} \cap V_{i} \neq \emptyset\right\} \subset V(\bar{x}) .
$$

Let $\left\{\lambda_{i}\right\}_{i=1}^{k}$ be a partition of unity subordinate to $\mathcal{V}$. Set $\Omega_{\varepsilon}:=\bigcup_{i=1}^{k} V_{i}$. Define $f: \Omega_{\varepsilon} \rightarrow E$ by

$$
f(x):=\sum_{i=1}^{k} \lambda_{i}(x) y_{i},
$$

where $x_{i} \in V_{i} \cap A$ and $y_{i}:=y_{x_{i}}$. Of course, $f$ is Lipschitz. Moreover, by the convexity of the values of $\Psi, f$ is a selection of $\Psi$ on $A$.

Let $x \in \bigcup_{i=1}^{k} V_{i}$. Since $\mathcal{V}$ is a star-refinement of $\{U(x)\}_{x \in A}$, there is $\bar{x} \in A$ such that $x, x_{i} \in U(\bar{x})$ for each $i \in\{1, \ldots, k\}$ with $x \in V_{i}$. Therefore, $y_{i} \in$ $F\left(x_{i}\right) \subset F(\bar{x})+(\varepsilon / 2) B_{1}$, and since $F(\bar{x})$ is convex, $f(x) \in F(\bar{x})+(\varepsilon / 2) B_{1}$.

Hence, $f(x) \in F(B(x, \varepsilon))+\varepsilon B_{1}$ and the proof is complete.

The lemmas above allow us to prove the following result on equilibria.

Proposition 3.8. Let $K=\overline{\operatorname{Int} K} \subset \mathbb{R}^{n}$ be a Lefschetz set with $\chi(K) \neq 0$ such that the Clarke cone map $\Psi(\cdot):=C_{\widehat{K}}(\cdot)$ is l.s.c. on $\widehat{K}$. Assume that $\Omega \subset \mathbb{R}^{n}$ is an open neighbourhood of $K$ and $F: \Omega \multimap \mathbb{R}^{n}$ a Marchaud map satisfying (17) on $\widehat{K} \cap \Omega$. Then $F$ has an equilibrium in $K$. 
REMARK 3.9. It can be proved that these assumptions imply that $K$ is viable under $-F$. However, here we relax the regularity assumption on $K$, compared with [3].

Proof. Let $\varepsilon>0$ be given. By Lemma 3.7, there exist an open neighbourhood $\Omega_{\varepsilon}$ of $\partial K$ in $\Omega$ and a Lipschitz map $f: \Omega_{\varepsilon} \rightarrow \mathbb{R}^{n}$ such that $f$ is an $\varepsilon$-approximation of $F$ on $\Omega_{\varepsilon}$ and a selection of $\Psi$ on $\partial K$.

If $K \backslash \Omega_{\varepsilon} \neq \emptyset$, then for an open set $V$ with $\partial K \subset V \subset \bar{V} \subset \Omega_{\varepsilon}$, we define an Urysohn function $v: \mathbb{R}^{n} \rightarrow[0,1]$ by

$$
v(x):=\max \left\{0,1-\frac{1}{\operatorname{dist}\left(\bar{V}, K \backslash \Omega_{\varepsilon}\right)} d_{\bar{V}}(x)\right\} .
$$

Now we can take any Lipschitz $\varepsilon$-approximation $g$ of $F$ on $K$, which exists according to [7], and join $f$ to $g$ by

$$
f_{\varepsilon}(x):=v(x) f(x)+(1-v(x)) g(x) .
$$

In this way we have obtained a Lipschitz map which is equal to $f$ on $\partial K$ and $f_{\varepsilon}(x) \in \operatorname{conv} F(B(x, \varepsilon))+\varepsilon B_{1}$ for every $x \in K \cup \Omega_{\varepsilon}$.

We show that $K$ is invariant under $-f_{\varepsilon}$. First, let $x$ be a solution for $-f_{\varepsilon}$ starting from an interior point of $K$. Suppose, on the contrary, that $x$ leaves $K$ at some $s>0$. Then $y(\cdot):=x(s-\cdot)$ is a solution for $f_{\varepsilon}$ starting from $x(s) \in \partial K$ and reaching the interior point of $K$ at $s>0$. But this is impossible since $\widehat{K}$ is invariant under $f_{\varepsilon}$. By continuity of the solution map $S_{-f_{\varepsilon}}$ the set $K$ is invariant under $-f_{\varepsilon}$.

Since $\varepsilon$ was arbitrary, we can choose a sequence $\varepsilon_{m} \rightarrow 0$ and $f_{m}:=f_{\varepsilon_{m}}$. Using Lemma 3.6, we can find for every $m \geq 1$ a point $x_{m} \in K$ with $0=f_{m}\left(x_{m}\right)$. By standard arguments, since $F$ has a closed graph, we pass to the limit as $m \rightarrow \infty$ obtaining an equilibrium of $F$ in $K$.

Corollary 3.10. Let $K=\overline{\operatorname{Int} K} \subset \mathbb{R}^{n}$ be a Lefschetz set with $\chi(K) \neq 0$ such that the map $\Psi(\cdot):=C_{\widehat{K}}(\cdot)$ is l.s.c. on $\widehat{K}$. Assume that $\Omega \subset \mathbb{R}^{n}$ is an open neighbourhood of $K$ and $F: \Omega \multimap \mathbb{R}^{n}$ a Marchaud map satisfying

$$
F(x) \cap \operatorname{Int} \Psi(x) \neq \emptyset \quad \text { for each } x \in \widehat{K} \cap \Omega \text {. }
$$

Then $F$ has an equilibrium in $K$.

Proof. It is easy to check that ocndition (18) implies (17).

EXAMPLE 3.11. Let

$$
\begin{aligned}
K_{1} & :=\left\{(x, y) \in \mathbb{R}^{2} \mid-1 \leq x \leq 0 \text { and } \sqrt{1-(x+1)^{2}}-1 \leq y \leq 1\right\}, \\
K_{2} & :=\left\{(x, y) \in \mathbb{R}^{2} \mid 0 \leq x \leq 1 \text { and } \sqrt{1-(x-1)^{2}}-1 \leq y \leq 1\right\}, \\
K & :=K_{1} \cup K_{2} \cup \operatorname{cl} B((1,1), 1) \cup \operatorname{cl} B((-1,1), 1) .
\end{aligned}
$$




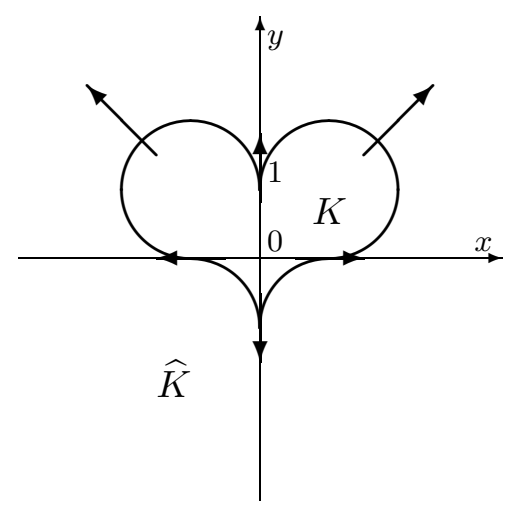

Consider any Marchaud map $F: \mathbb{R}^{2} \multimap \mathbb{R}^{2}$ with $x \in F(x)$ on $\partial K$. One can readily check that condition (17) is satisfied, as are the other assumptions of Theorem 3.8. Notice that neither $K$ nor $\widehat{K}$ is an $\mathcal{L}$-retract, so the approach of [3] cannot be used.

REMARK 3.12. Note that the class of sets such that the Clarke tangent cone map is l.s.c. contains the class of sleek sets, i.e., where the Bouligand cone map is l.s.c., so it contains all proximate retracts. One can easily see that this class is essentially larger. Note also that it is not included in the class of $\mathcal{L}$-retracts (the example above) and vice-versa (one can modify an example in $[19$, p. 219]).

Example 3.13. Consider the set $K$ from Example 3.11 and the following problem:

$$
\left\{\begin{array}{l}
\dot{x}(t)=f(x(t), u(t), v(t)), \\
x(0)=x_{0} \in K, \\
u, v \in U
\end{array}\right.
$$

where $U=\operatorname{cl} B(0, r), r<1$. Assume that $f(x, u, v)=h(x, u)+g(x, v)$, where $h, g: \mathbb{R}^{2} \rightarrow \mathbb{R}^{2}$ are continuous maps satisfying

$$
\begin{gathered}
h(x, u)=x+u \quad \text { for every } x \in \partial K, \\
|g(x, v)| \leq l|x|, l<r, \quad \text { for each } x \in \partial K \text { and } v \in U,
\end{gathered}
$$

and $g(\{x\} \times U)$ is convex for every $x \in \mathbb{R}^{n}$.

Then there are $x_{0} \in \mathbb{R}^{n}$ and controls $u$ and $v$ giving a stationary trajectory for (19). Indeed, one can check that $F(x):=f(\{x\} \times U \times U)$ satisfies the assumptions of Theorem 3.8.

4. Strict equilibria. In this section we find several sufficient conditions for existence of strict equilibria in prescribed compact sets. 
4.1. Basic properties. The notion of a strict equilibrium coincides with the one of ordinary equilibrium in the single-valued case while it brings important information for multivalued maps and differential inclusions. For instance, from each strict equilibrium of a Lipschitz map there starts only a stationary trajectory.

Some difficulties in finding strict equilibria are visible in the following example.

Example 4.1. Consider the set $K:=[-2,2] \times[-2,2]$ and the Lipschitz $\operatorname{map} F: \mathbb{R}^{2} \multimap \mathbb{R}^{2}$,

$$
F(x, y):=[x-1, x+1] \times\{0\} .
$$

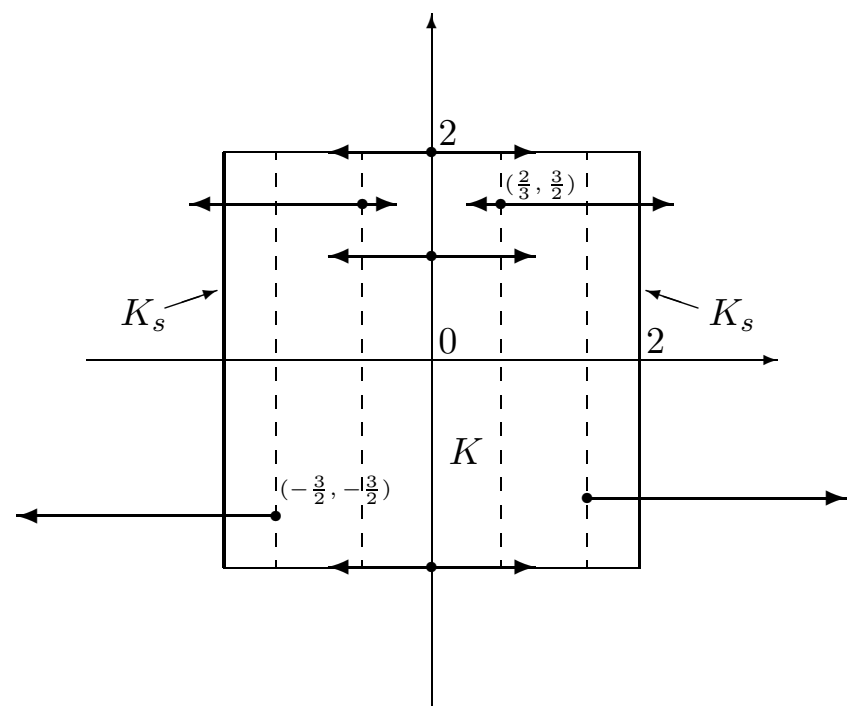

It is easy to check that $K_{s}=K_{e}=(\{-2\} \times[-2,2]) \cup(\{2\} \times[-2,2]), K_{s}$ is compact and disconnected with $\chi\left(K, K_{s}\right)=-1 \neq 0$. Notice that the behaviour of $F$ on $\partial K$ guarantees the existence of equilibria. Nevertheless, none of them is strict.

One of possible methods of finding strict equilibria is to study singlevalued selections of the multivalued map, as in the following general observation.

REMARK 4.2. Let $K \subset X$ be a compact set and $F: K \multimap \mathbb{R}^{n}$ a multivalued map. Assume that there is a single-valued selection $f: K \rightarrow \mathbb{R}^{n}$ of $F$ with

$$
\forall x \in K: \quad f(x)=0 \Rightarrow F(x)=\{0\} .
$$

Then, obviously, each equilibrium of $f$ is a strict equilibrium of $F$. 
However, it is not obvious how to find, for a given multivalued map, a selection satisfying (20) and simultaneously having an equilibrium.

Below we give several conditions implying the existence of such a selection.

Proposition 4.3. Let $K \subset \mathbb{R}^{n}$ be a compact set, $\Omega$ an open neighbourhood of $K$ in $\mathbb{R}^{n}$ and $F: \Omega \multimap \mathbb{R}^{n}$ a Lipschitz map satisfying (3). Assume that the map $f:=\sigma(F(\cdot))$, where $\sigma(F(x))$ is a Steiner point of $F(x)$, satisfies assumptions guaranteeing the existence of equilibria of $f$. Then there exists a strict equilibrium of $F$ in $K$.

Proof. Notice that $f$ is a Lipschitz selection of $F$ satisfying (20).

Below we give sufficient conditions for (3) to hold.

Corollary 4.4. If instead of (3) we assume that

$$
F(x) \cap-F(x) \subset\{0\} \quad \text { for every } x \in K,
$$

(this can be expressed by saying that no value of $F$ contains opposite directions) or

$$
\begin{aligned}
& \text { there are a map } \gamma: K \rightarrow \partial B_{1} \text { and a constant } c>0 \text { such that } \\
& \langle\gamma(x), y\rangle \geq c|y| \text { for every } x \in K \text { and } y \in F(x)
\end{aligned}
$$

(that is, there is a guiding function for $F$ ), then there exists a strict equilibrium of $F$ in $K$.

We also list some sufficient conditions guaranteeing the existence of equilibria for all possible continuous (or Lipschitz) selections of $F$.

Example 4.5. (1) Let $K$ and $F$ satisfy the assumptions of Proposition 3.4 or Corollary 3.2. Then each Lipschitz selection of $F$ has an equilibrium in $K$.

(2) Let $K$ be a closed ball in $\mathbb{R}^{n}$ and $F: K \multimap \mathbb{R}^{n}$ be of the form $F(x)=x-\varphi(x)$, where $\varphi$ is a continuous, compact convex valued map satisfying

$$
x \notin \lambda \varphi(x) \quad \text { for each } x \in \partial K \text { and } 0<\lambda<1 .
$$

Then each continuous selection (existing due to Michael's selection theorem) has an equilibrium in $K$.

(3) Let $K=\overline{\operatorname{Int} K}$ be compact and $F: K \multimap \mathbb{R}^{n}$ a Lipschitz, compact convex valued map with $0 \notin F(\partial K)$. If $\operatorname{Deg}(F, \operatorname{Int} K) \neq 0$, then each continuous selection has an equilibrium in $K$.

The second statement follows easily from the Nonlinear Alternative applied to any continuous selection of $\varphi$ (see e.g. [12]). The third one is an immediate consequence of a construction of the topological degree for compact convex valued u.s.c. maps (see e.g. [8]). 
As a consequence, the following fixed point result can be obtained.

Corollary 4.6. Let $K$ be a closed ball in $\mathbb{R}^{n}$ and $\varphi: K \multimap \mathbb{R}^{n} a$ Lipschitz map with compact strictly convex values which satisfies (23) and

$$
x \notin \varphi(x) \backslash \operatorname{ext} \varphi(x) \quad \text { for every } x \in K,
$$

where ext $\varphi(x)$ stands for the set of extremal points of $\varphi(x)$. Then there is a point $x \in K$ such that $\varphi(x)=\{x\}$.

Proof. Note that the map $F(\cdot):=\cdot-\varphi(\cdot)$ satisfies (21) and use Example 4.5(2).

4.2. Strict equilibria on smooth sets. In the present subsection, under some smoothness assumptions on $K$, we obtain strict equilibria of a Lipschitz map $F$ without looking for a Lipschitz selection of $F$ satisfying (20). Moreover, although the existence of a strict equilibrium implies the existence of equilibria of each selection, in the situation presented below $\left(K_{s} \neq K_{e}\right)$ it would not be easy to check it for any of them.

This subsection is devoted to the proof of the second main result of our paper, Theorem 1.2. Note that the assumption $0 \notin F(x)$ on $\partial K$ is essential. To see this, consider the following.

ExAmple 4.7. Let $K:=[0,1] \subset \mathbb{R}$ and $F(x)=[-1,0]$. Then $K_{s}=\emptyset$, so $\chi\left(K, K_{s}\right)=1 \neq 0$. Obviously, conditions (I), (III) and (3) are satisfied while there is no strict equilibrium of $F$ in $K$.

In what follows we proceed analogously to Subsection 3.1.

Proof of Theorem 1.2. We modify the proof of Lemma 3.3 and find first an open set $U \subset \mathbb{R}^{n}$ with $K \backslash K_{s}(F) \subset U, K_{s}(F) \cap U=\emptyset$ and a Lipschitz map $g: U \rightarrow \mathbb{R}^{n}$ satisfying (A), (C), (D) and

(E) for every $x \in \operatorname{Int} K$, if $g(x)=0$, then $\sigma(F(x))=0$.

To do this, we repeat Step 1, Case 1 and Case 2 of the proof of Lemma 3.3 , obtaining $v_{x}(y):=v_{x}$ for $y \in U_{x}$ in both cases.

In Case 3, that is, for $x \in \operatorname{Int} K$ with $0 \in F(x)$, take an open neighbourhood $U_{x} \subset B(x, \varepsilon)$ in $\mathbb{R}^{n}$ such that $U_{x} \cap \partial K=\emptyset$, and for each $y \in U_{x}$, put $v_{x}(y):=\sigma(F(y))$. Then, obviously, $v_{x}(y) \in F(y) \subset F(y)+d_{K_{s}(F)}(y) B_{1}$ for every $y \in U_{x}$.

Choosing a countable, locally finite covering $\left\{U_{x_{i}} \mid x_{i} \in K \backslash K_{s}(F)\right\}$ of $K \backslash K_{s}(F)$ and a subordinate smooth partition of unity $\left\{\lambda_{i}\right\}$, we can define $g: U=\bigcup_{i=1}^{\infty} U_{x_{i}} \rightarrow \mathbb{R}^{n}$ by

$$
g(x):=\sum_{i \in I(x)} \lambda_{i}(x) v_{x_{i}}(x) .
$$

Since $g$ is Lipschitz and satisfies (A), (C), (D), we have to show only (E). 
We claim that for every $x \in \operatorname{Int} K$, if $g(x)=0$, then $0 \in F\left(x_{i}\right)$ for each $i \in I(x)$ and $x_{i} \in \operatorname{Int} K$.

Indeed, otherwise there is $i \in I(x)$ with $0 \notin F\left(x_{i}\right)$. From the construction of $U_{x_{i}}$ it follows that $0 \notin F(x)$. Therefore,

$$
0=g(x) \in F(x)+\frac{1}{2} d_{F(x)}(0) \not \supset 0 ;
$$

a contradiction. Since there are no equilibria of $F$ on $\partial K$, we have $x_{i} \in \operatorname{Int} K$.

Since $0 \in F\left(x_{i}\right)$ and $x_{i} \in \operatorname{Int} K$, by the construction of $v_{x_{i}}(\cdot)$ one has $v_{x_{i}}=\sigma(F(x))$ for every $i \in I(x)$. Hence, $g(x)=\sigma(F(x))$.

Now, following Step 2 of the proof of Lemma 3.3, we construct a suitable map $f$ satisfying (a)-(d). Moreover, from the construction of $U_{x_{i}}$ it follows that if $f(x)=0$, then $\beta_{i}(x)=0$ for every $i \geq 1$, which implies that

$$
0=f(x)=g(x)=\sigma(F(x)) \text {. }
$$

Applying Proposition 3.1 for $f$ we find an equilibrium $x \in \operatorname{Int} K$ of $f$. By (25) and (3), it is a strict equilibrium of $F$; the proof is complete.

5. Appendix. Below we give conditions, in terms of tangent cones, allowing us to check when a point $x \in \partial K$ belongs to $K_{s}$. As a consequence, we obtain a sufficient condition for $K_{s}$ to be closed. We will use the following notation (cf. [18]):

$$
K_{o}:=\left\{x \in \partial K \mid F(x) \cap T_{K}(x)=\emptyset\right\}, \quad D_{K}(x):=\mathbb{R}^{n} \backslash T_{\overline{\mathbb{R}^{n} \backslash K}}(x) .
$$

The relation between $K_{s}$ and $K_{o}$ is given in the following.

Lemma 5.1. For any Marchaud map,

$$
K_{o} \subset K_{s} \subset \bar{K}_{o} .
$$

Proof. If a point belongs to $K_{o}$, then any solution starting from this point leaves $K$ immediately, otherwise the tangential condition (2) would be satisfied. So, $K_{o} \subset K_{s}$.

Let us now show that $K_{s} \subset \bar{K}_{o}$. The set $K \backslash \bar{K}_{o}$ is locally compact, and the tangential condition (2) is everywhere satisfied from the very definition of $K_{o}$. The viability theorem (cf. [1, Theorem 3.3.2]) states that for any point of $K \backslash \bar{K}_{o}$, there exists at least one solution starting from this point, which remains in $K$ on some $[0, \tau], \tau>0$, i.e., the point is not in $K_{s}$. This proves that if a point does not belong to $\bar{K}_{o}$, then it does not belong to $K_{s}$, i.e., $K_{s} \subset \bar{K}_{o}$.

Unfortunately, the set $K_{o}$ is seldom closed. We will now study what happens for points of $\bar{K}_{o} \backslash K_{o}$. A proof of the lemma below is given for the convenience of the reader because it has not been published anywhere yet.

Lemma 5.2 ([5, Proposition 3.1] or [6, Proposition 2.1]). Let $K$ be closed and $F$ be a Marchaud map locally Lipschitz around $x \in \bar{K}_{o} \backslash K_{o}$. 
(1) If $F(x) \cap\left(D_{K}(x) \cup T_{\partial K \backslash K_{o}}(x)\right)=\emptyset$, then $x \in K_{s}$.

(2) If $F(x) \cap D_{K}(x) \neq \emptyset$ or $F(x) \cap T_{K_{o}}(x)=\emptyset$, then $x \notin K_{s}$.

Proof. To prove (1) assume for a while that $x \notin K_{s}$, i.e., that there is $x(\cdot) \in S_{F}(x)$ viable in $K$ on $[0, \varepsilon]$ with $\varepsilon>0$.

If there exists a sequence $t_{m}>0$ converging to 0 such that $x\left(t_{m}\right) \in \partial K$ for any $m$, then, up to a subsequence, and because $x\left(t_{m}\right) \notin K_{o}$ from the viability theorem, we have

$$
\frac{x\left(t_{m}\right)-x}{t_{m}} \rightarrow v \in F(x) \cap T_{\partial K \backslash K_{o}}(x),
$$

which is impossible.

So, we can find $\alpha>0$ such that $x(s) \in \operatorname{Int} K$ for $0<s \leq \alpha$. Recall that $x \notin K_{o}$ means that $F(x) \cap T_{K}(x) \neq \emptyset$. On the other hand, from the assumption, $F(x) \cap D_{K}(x)=\emptyset$. So, by (15), there is $v \in F(x) \cap T_{\partial K}(x)$. Since $F(x) \cap T_{\partial K \backslash K_{o}}(x)=\emptyset$, one can find $h_{m} \rightarrow 0$ and $v_{m} \rightarrow v$ such that $x+h_{m} v_{m} \in K_{o}$. According to Filippov's theorem (see e.g. [1]), there exist $x_{m}(\cdot) \in S_{F}\left(x+h_{m} v_{m}\right)$ converging to $x(\cdot)$ in the topology of uniform convergence. Moreover, $x_{m}(\cdot)$ leaves $K$ immediately for any $m$ because $x+$ $h_{m} v_{m} \in K_{o} \subset K_{s}$. Since $x(\alpha) \in \operatorname{Int} K$, also $x_{m}(\alpha) \in \operatorname{Int} K$ for $m$ large enough, and there is $t_{m} \in(0, \alpha)$ such that $x_{m}(\cdot)$ is viable on $\left[t_{m}, \alpha\right]$. We choose $t_{m}$ such that $x_{m}\left(t_{m}\right) \in \partial K$, and in fact $x_{m}\left(t_{m}\right) \in \partial K \backslash K_{o}$. Since $x(s) \in \operatorname{Int} K$ for $0<s \leq \alpha$, the sequence $t_{m}$ converges to 0 .

Let us now remark that from the convexity of $F(x)$, up to a subsequence,

$$
\frac{x_{m}\left(t_{m}\right)-\left(x+h_{m} v_{m}\right)}{t_{m}} \rightarrow w \in F(x) .
$$

Indeed, for any $\varepsilon>0$, there exists a neighbourhood $V$ of $x$ on which $F(y) \subset$ $F(x)+\varepsilon B_{1}$, because $F$ is upper semicontinuous. For $m$ large enough, $x_{m}(\cdot)$ remains in $V$ on $\left[0, t_{m}\right]$, and we have

$$
x_{m}\left(t_{m}\right)-\left(x+h_{m} v_{m}\right) \in t_{m}\left(F(x)+\varepsilon B_{1}\right),
$$

which proves $(26)$.

Now we show that $\frac{x_{m}\left(t_{m}\right)-x}{t_{m}+h_{m}}$ converges, up to a subsequence, to a point of $F(x) \cap T_{\partial K \backslash K_{o}}(x)$, which contradicts our assumption.

To this end, notice that

$$
\frac{x_{m}\left(t_{m}\right)-x}{h_{m}+t_{m}}=\frac{t_{m}}{t_{m}+h_{m}} \frac{x_{m}\left(t_{m}\right)-\left(x+h_{m} v_{m}\right)}{t_{m}}+\frac{h_{m}}{t_{m}+h_{m}} v_{m}
$$

which converges, up to a subsequence, to $\lambda w+(1-\lambda) v$, where $\lambda \in[0,1]$. We conclude thanks to the convexity of $F(x)$.

To prove (2), we have to show that there is $x(\cdot) \in S_{F}(x)$ which remains in $K$ on some $[0, \varepsilon]$ with $\varepsilon>0$. 
If $F(x) \cap D_{K}(x) \neq \emptyset$, Filippov's Theorem shows that there exists a solution in $S_{F}(x)$ locally viable in $K$.

If not, since $x \notin K_{o}$, the set $F(x) \cap T_{\partial K}(x)$ is not empty. Since $F(x) \cap$ $T_{K_{o}}(x)=\emptyset$, there exists $v \in F(x) \cap T_{\partial K \backslash K_{o}}(x)$. Let $v_{m} \rightarrow v$ and $h_{m} \rightarrow 0$ be such that $x+h_{m} v_{m} \in \partial K \backslash \bar{K}_{s}$ (recall that $\bar{K}_{s}=\bar{K}_{o}$ ). Therefore there exist $x_{m}(\cdot) \in S_{F}\left(x+h_{m} v_{m}\right)$ viable in $K$ on $\left[0, t_{m}\right]$, where $t_{m} \in(0, \infty]$. We choose $x_{m}(\cdot)$ and $t_{m}$ in such a way that

$$
\begin{aligned}
t_{m} & =\sup \left\{\inf \{t>0 \mid x(t) \notin K\} \mid x(\cdot) \in S_{F}\left(x+h_{m} v_{m}\right)\right\} \\
& =\inf \left\{t>0 \mid x_{m}(t) \notin K\right\} .
\end{aligned}
$$

Since $F$ is a Marchaud map, such $t_{m}$ and $x_{m}$ exist (see [1, p. 135]). It is seen that if $t_{m}<\infty$, then $x_{m}\left(t_{m}\right) \in K_{s}$.

Assume that a subsequence of $\left\{t_{m}\right\}$, called $\left\{t_{m}\right\}$ again, converges to 0 . Then, as in the first part of the proof, we can show that a subsequence of $\frac{x_{m}\left(t_{m}\right)-x}{t_{m}+h_{m}}$ converges to an element of $F(x) \cap T_{K_{s}}(x)$, i.e., to an element of $F(x) \cap T_{K_{o}}(x)$. This is impossible.

So, there exists $\varepsilon>0$ such that each $x_{m}(\cdot)$ is viable in $K$ on $[0, \varepsilon]$. A subsequence of $\left\{x_{m}(\cdot)\right\}$ converges to an element $x(\cdot) \in S_{F}(x)$ which remains in $K$ on $[0, \varepsilon]$, i.e., $x$ does not belong to $K_{s}$. This ends the proof.

\section{References}

[1] J.-P. Aubin, Viability Theory, Birkhäuser, Boston, 1991.

[2] J.-P. Aubin and H. Frankowska, Set-Valued Analysis, Birkhäuser, Boston, 1990.

[3] H. Ben-El-Mechaiekh and W. Kryszewski, Equilibria of set-valued maps on nonconvex domains, Trans. Amer. Math. Soc. 349 (1997), 4159-4179.

[4] R. Brown, The Lefschetz Fized Point Theorem, Scott, Foresman and Co., Glenview, IL, 1971.

[5] P. Cardaliaguet, Sufficient conditions of nonemptiness of the viability kernel, $\mathrm{PhD}$ thesis, Chapter 8, Univ. Paris IX Dauphine, 1992.

[6] -, Conditions suffisantes de non-vacuité du noyau de viabilité, C. R. Acad. Sci. Paris Sér. I 314 (1992), 797-800.

[7] A. Cellina, A theorem on the approximation of compact multivalued mappings, Atti Accad. Naz. Lincei Rend. 8 (1969), 149-153.

[8] A. Cellina and A. Lasota, A new approach to the definition of topological degree for multi-valued mappings, ibid. 6 (1970), 434-440.

[9] F. Clarke, Yu. S. Ledyaev and R. J. Stern, Fixed points and equilibria in nonconvex sets, Nonlinear Anal. 25 (1995), 145-161.

[10] B. Cornet, Paris avec handicaps et théorèmes de surjectivité de correspondances, C. R. Acad. Sci. Paris Sér. A 281 (1975), 479-482.

[11] - Euler characteristic and fixed-point theorems, Positivity 6 (2002), 243-260.

[12] J. Dugundji and A. Granas, Fixed Point Theory, Vol. I, Monografie Mat. 61, PWN, Warszawa, 1982.

[13] G. Gabor and M. Quincampoix, On existence of equilibria of set-valued maps, Boll. Un. Mat. Ital. B 6 (2003), 309-321. 
[14] L. Górniewicz, Topological Fixed Point Theory of Multivalued Mappings, Kluwer, Dordrecht, 1999.

[15] M. Mrozek, Periodic and stationary trajectories of flows and ordinary differential equations, Zesz. Nauk. Uniw. Jagielloń. 860, Acta Math. 27 (1988), 29-37.

[16] S. Plaskacz, On the solution sets of differential inclusions, Boll. Un. Mat. Ital. A (7) 6 (1992), 387-394.

[17] M. Quincampoix, Frontières de domaines d'invariance et de viabilité pour les inclusions différentielles avec contraintes, C. R. Acad. Sci. Paris 311 (1990), 411-416.

[18] —, Differential inclusions and target problems, SIAM J. Control Optim. 30 (1992), 324-335.

[19] R. T. Rockafellar and R. J.-B. Wets, Variational Analysis, Springer, 1998.

Laboratoire de Mathématiques

Université de Bretagne Occidentale

6, avenue Victor Le Gorgeu B.P. 809

29285 Brest Cedex, France

E-mail: Pierre.Cardaliaguet@univ-brest.fr

Marc.Quincampoix@univ-brest.fr
Faculty of Mathematics and Computer Science Nicolaus Copernicus University Chopina 12/18

87-100 Toruń, Poland E-mail: ggabor@mat.uni.torun.pl

Reçu par la Rédaction le 18.10.2001

Révisé le 22.1.2003 